Title: TRAPS FOR ANTIMATTER AND ANTIHYDROGEN PRODUCTION

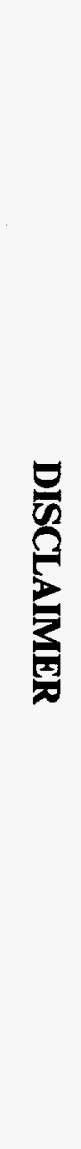

M. H. Holzscheiter

Trapped Charged Particles and Fundamental Physics Lyseuil, Sweden August 13-22, 1994

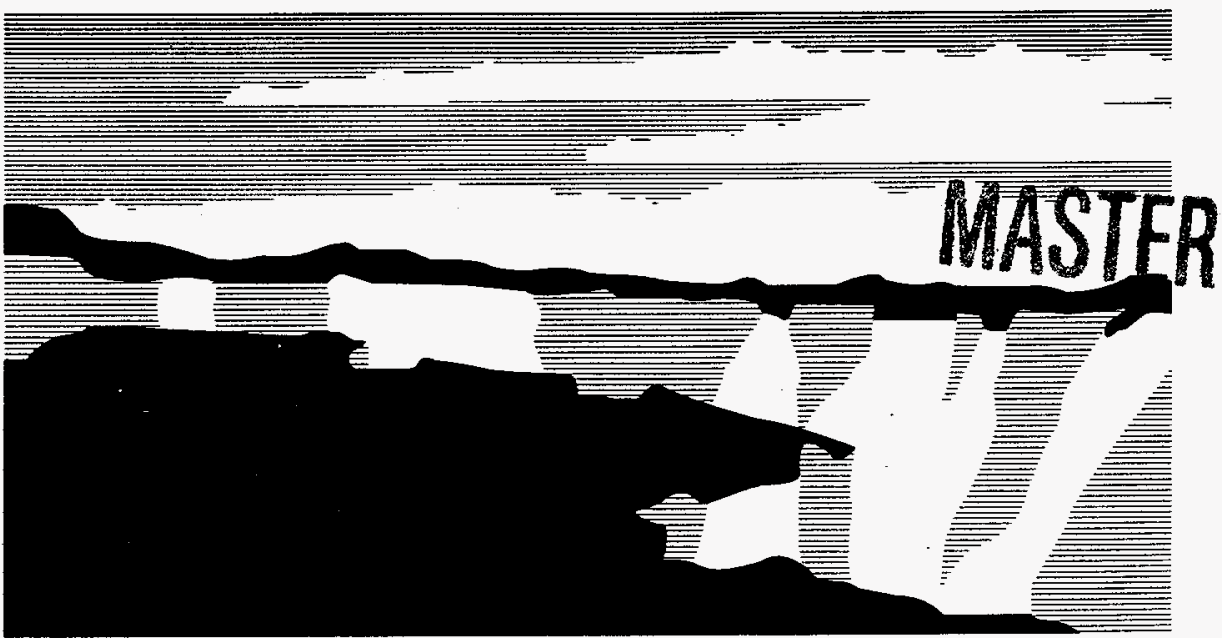

Los Alamos National Laboratory, an affirmative action/equal opportunity employer, is operated by the University of California for the U.S. Department of Energy under contract W-7405-ENG-36. By acceptance of this article, the publisher recognizes that the U.S. Government retains a nonexclusive, royalty-free license to publish or reproduce the published form of this contribution, or to allow others to do so, for U.S. Government purposes. The Los Alamos National Laboratory requests that the publisher identify this article as work performed under the auspices of the U.S. Department of Energy. 


\section{DISCLAIMER}

Portions of this document may be illegible in electronic image products. Images are produced from the best available original document. 


\title{
Traps for Antimatter and Antihydrogen Production
}

\author{
Michael H. Holzscheiter \\ Los Alamos National Laboratory \\ Los Alamos, NM 87545 \\ USA
}

\begin{abstract}
Even though positrons have been captured and stored in ion traps for precision measurements, the recent trapping and cooling of antiprotons may be considered as the beginning of a new era in antimatter research. For the first time all the ingredients to produce the first atom of the antimatter world, the antihydrogen atom, are at hand, and several groups have entered an active discussion on the feasibility of producing antihydrogen as well as on the possibility to perform precision tests on CPT and gravity. At the same time, the trapping of reasonable large numbers of antiprotons has opened up the way for a variety of exciting physics with ultra-low energy antiprotons, ranging from atomic physics issues to nuclear physics and medical applications. I will describe the current status of the work on trapping antiprotons and positrons, discuss possible physics applications of this technique, and describe the two most promising routes to produce antihydrogen for precision spectroscopy. Towards the end a few comments on storing the produced antihydrogen and on utilizing antihydrogen for gravity measurements and for CPT tests are given.
\end{abstract}




\section{Introduction.}

In recent years trapping of antimatter has made a giant leap forward due to the successes in trapping and cooling antiprotons to ultra-low energy $[1,2,3]$. Even though trapping of positrons had been accomplished some time ago [4,5], this may be viewed to define the beginning of a new era of antimatter physics, especially, since now for the first time we have all the ingredients to produce antihydrogen, the first complete atom of the antimatter world.

Even though not entirely necessary, the art and technique of trapping (both charged particles as well as neutral atoms) will play an important role in the field of antimatter research and the necessary ingredients are detailed further in this paper. Section 2 describes the current status on trapping of antiprotons and section 3 discusses possible applications of this technique to atomic, nuclear, and gravitational physics. Section 4 describes the different production mechanisms for antihydrogen and reviews the work on trapping positrons. In section 5 some speculations about trapping the produced antihydrogen for spectroscopy, CPT tests, and gravitational tests are presented.

\section{Trapping and cooling of antiprotons.}

Antiprotons are produced at very high energies, with the lowest energies at which they are delivered to physics users at this time being $5.9 \mathrm{MeV}$ at the Low Energy Antiproton Ring (LEAR) at CERN [6]. To further reduce this energy a number of methods have been proposed and partially tested: Deceleration by a Radio-FrequencyQuadrupole Accelerator (RFQ) operated in reversed mode [7], slowing down antiprotons in a dilute gas in the 'Anti-Cyclotron' [8], and degrading the antiproton energy by passing the beam through thin foils $[9,10]$. Matching the RFQ acceptance to the phase space of the LEAR output beam has been identified as a main problem of the first method, and an experiment using an RFQ to decelerate an antiproton beam from $2 \mathrm{MeV}$ to $200 \mathrm{keV}$ [11] has recently been abandoned. Initial tests of the Anti-Cyclotron approach have been performed, with current development continuing at the Paul Scherrer Institute (PSI) in Switzerland using muons [12]. The degrading foil method has been used by two experiments at LEAR with promising results during the last years [2,3], and I will discuss this method in some more detail.

When protons or antiprotons pass through matter they loose energy by collisions with the nuclei of the material. If the thickness of the material is increased particles 
eventually are stopped in the material, and, in the case of antiprotons, annihilate. Using the computer code TRIM91 [13], which is based on energy loss data for protons, one can calculate the expected linear density of material at which a maximum number of particles with low energy is transmitted, and how large the number for a specified energy bin should be. One finds this optimum thickness to be near the point where $50 \%$ of the incoming particles are transmitted. Adjustments for different stopping powers for protons vs. antiprotons (the Barkas Effect [14]) must be made empirically. These calculations predict as much as $5 \%$ of the incoming antiprotons being transferred into an energy bin of 0 to 50 $\mathrm{keV} \mathrm{[10].} \mathrm{At} \mathrm{this} \mathrm{energy} \mathrm{it} \mathrm{is} \mathrm{possible} \mathrm{to} \mathrm{electro-magnetically} \mathrm{capture} \mathrm{the} \mathrm{antiprotons} \mathrm{in} \mathrm{a}$ Penning trap and to further reduce their temperature using electron cooling [15], stochastic cooling [16], or resistive cooling [17].

This method has been successfully used by experiment PS196 [2] with a capture efficiency of $2 \times 10^{-4}$ per $\mathrm{keV}$ well depth. More than 20000 antiprotons have been captured into a small Penning trap and cooled to temperatures below $100 \mathrm{meV}$. Observed cooling times were approximately 10 seconds. Energy widths as small as $9 \mathrm{meV}$ have been directly observed by releasing the trapped antiprotons from the trap. After the antiprotons are cooled, the trap well can be lowered again to accept a new pulse of antiprotons into the same trap. This method of "stacking" has been demonstrated by the PS196 team and approximately 100,000 cold antiprotons have been captured into the Penning trap utilizing about 10 consecutive pulses from LEAR.

\subsection{Trapping of $30 \mathrm{keV}$ antiprotons from LEAR.}

In order to determine the ultimate efficiency that can be achieved for degrading and capturing antiprotons in a Penning trap, the first part of the PS200 experimental setup was installed at LEAR [3]. This part consists of a Penning type trap of $50 \mathrm{~cm}$ total length and $3.8 \mathrm{~cm}$ diameter, situated in the horizontal, cryogenic bore of a super conducting magnet capable of producing a magnetic field of up to 6 Tesla. Figure 1 shows the general layout of the experiment and the different detectors used to monitor the incoming beam and to verify the capture of antiprotons.

A particle pulse from LEAR is transported to the front end of the experiment, which contains a parallel plate avalanche counter (PPAC) for beam monitoring. After exiting the LEAR beam line through a 12 micron titanium window, passing through the PPAC chambers, a gas cell for fine tuning of the energy degrading, and entering the vacuum system of the experiment through another 12 micron titanium window, the beam 
energy has been reduced to approximately $3.7-4.0 \mathrm{MeV}$. Due to the transverse scattering in the material the particles have to pass through, a relatively large angular spread is introduced into the beam, which is then focused by the fringe magnetic field of the super conducting magnet. By choosing a specific magnetic field strength for the particular energy of the incoming beam a focal point can be placed onto the entrance foil of the trap. In this 135 micron gold coated aluminum foil the antiprotons will loose energy by collisions with the atoms of the foil material and, assuming proper adjustment of the additional degrader material upstream, an optimum number of low energy particles will exit from the downstream face of the foil. These particles will be reflected by the electrical potential at the far end of the trap and travel back towards the entrance electrode. This electrode is rapidly ramped up to potential before the particles can escape, thereby capturing them within the volume of the trap.

The principal design parameter for the trap is the length necessary to capture particles of energies up to $30 \mathrm{keV}$ emerging from this foil during a LEAR pulse of typically $200 \mathrm{~ns}$ duration. At a $1 \mathrm{~m}$ round trip distance, the time remaining after the last particle has entered the trap before the first particle is reflected back to the entrance is 220 ns for a $30 \mathrm{kV}$ well depth. Our current $30 \mathrm{kV}$ pulser has a $125 \mathrm{~ns}$ rise, allowing a total of $95 \mathrm{~ns}$ for jitter and uncertainty in the trigger timing.

According to these values we have constructed a trap structure which consists of 7 electrodes: the entrance foil, a central region comprised of five cylinders ( 2 endcaps, 2 compensation electrodes, and the central ring) with lengths and diameters carefully chosen to produce a harmonic, orthogonalized, quadrupole potential in the central region [18], and a cylindrical high voltage exit electrode. For the purpose of the initial antiproton capture the trapping region is defined by the entrance foil and the high voltage exit electrode. This region of the trap has no harmonic properties and will be referred to as the "catching trap" throughout this paper. The central, harmonic region serves a dual purpose: to initially hold cold electrons in preparation for the electron cooling, and then to collect the cooled antiprotons after the electron cooling has taken place. This trap is instrumented with two independent tuned circuits for the detection of electrons and antiprotons via the signals induced in the compensation rings and for resistively cooling the antiprotons after the electrons have been ejected.

To establish the capture of low energy antiprotons, they are released from the trap after a predetermined storage time and detected by the annihilation of pbars on the surface of the microchannel plate detector (MCP) using scintillators outside the magnet dewar as 
well as by direct counts from the MCP. To reduce the background rate in the 'hot' accelerator environment the scintillators are used in a 2-fold coincidence set-up and can additionally be gated by the MCP pulses. The detection efficiency has been deduced from Monte Carlo calculations [3] to be $7 \%$, a value which has been experimentally confirmed using a slow, continuous spill from LEAR. The release is accomplished by lowering the potential of the down stream end-cap of the trap linearly with a time constant large compared to the oscillation period of the particles in the trap. Particles will escape from the trap when their kinetic energy is greater than the potential barrier. This generates a time-of-arrival spectrum which reflects the energy distribution of the particles in the trap prior to the release. Figure 2 shows such an energy spectrum of approximately 500,000 antiprotons released from the trap $500 \mathrm{msec}$ after the pulser had fired to capture a pulse delivered from LEAR to our experiment.

To measure the storage time of antiprotons in our trap, which is not housed in a hermetically sealed, cryogenic vacuum system as the PS196 experiment is, but which is strongly coupled to a room temperature vacuum system at a base pressure near $10^{-10} \mathrm{Torr}$, the delay time between capture and release is varied. The number of detected antiprotons for each of these "shots" is normalized to the total intensity reading from the NE1 10 beam monitor and the results are plotted in figure 3 . Fitting the first 11 data points to an exponential decay yields a lower storage time limit of approximately 30 minutes. Including the last data point would reduce this time to 3 minutes, but this data point deserves special attention, as explained in the next section.

\subsection{Electron cooling of antiprotons.}

During the storage time measurements a noticeable change in the spectral shape could be noticed. After storage times of typically around 15-20 seconds, high energy particles could no longer be observed, and the energy distribution had started to shift towards later channels in the release spectrum without a decrease in the total number of particles. After 30 - 40 seconds all counts in the arrival time spectrum where concentrated at energies below $1 \mathrm{keV}$. Figure 4 shows a selection of energy spectra for 3 different storage times $(8,20$, and 70 seconds). In Figure 5 the shift of the centroid of the energy distribution is plotted vs time and fitted to a single exponential, yielding a cooling time constant of approximately 16 seconds. For the number of antiprotons in the trap, such a cooling time requires an electron density of approximately $10^{8}$ electrons $/ \mathrm{cm}^{3}$ [15]. As later tests revealed, electrons were produced by field emission from sharp points on the trap electrodes as well as by impact of the primary beam onto the degrader foil. These 
electrons are stored inside the well, cool rapidly by synchrotron radiation and eventually collect in the central part of the trap. To gain better control over this cooling process we have installed an electron source, consisting of a hot filament and appropriate extraction and focusing electrodes, in the fringe magnetic field region. Electrons from this source are allowed to impinge onto the inside of the degrading foil, thereby releasing neutral molecules, which can in turn be ionized by the electron beam electrons. Secondary electrons produced via this mechanism will be trapped and cooled by synchrotron radiation and will be collected in the central well. Using both a resonant detection technique as well as extracting these electrons from the central well to the MCP, we established that we can load the inner well with $10^{8}$ electrons using a primary electron beam of $50 \mu \mathrm{A}$ for $10-30$ seconds.

Antiprotons oscillating in the large catching trap interact via Coulomb interaction with these electrons and dissipate energy into the electron cloud, which in turn is continuously cooled by synchrotron radiation, until both electron and antiproton clouds arrive at a thermal distribution in equilibrium with the ambient temperature of the apparatus (approximately 25 Kelvin in the tests described here). Such a low temperature could not be observed under current experimental conditions, since the electrons are not ejected before the antiprotons are released, and the cloud experiences a (violent) Coulomb explosion when the trapping fields are lowered. Nevertheless, in the spectrum observed after 70 seconds storage time, the majority of the counts are concentrated in five channels, representing an apparent width of less than $100 \mathrm{eV}$. The ultimate energy width (obtainable by ejecting the electrons beforehand and releasing the antiprotons more slowly) is expected to be much lower, and will depend on the detailed mechanism chosen for the ejection of the antiproton bunches.

\subsection{Ejection of antiprotons from the PS200 catching trap.}

For most experiments envisioned at this time, the antiprotons will need to be ejected from the trap once the initial cooling has taken place. For the gravity measurement proposed in PS200 all antiprotons will be transferred in a single bunch into a small Penning trap at the bottom of the vertical time-of-flight experiment. Here they will be resistively cooled to $4.2 \mathrm{~K}$ and then released in bunches containing approximately 100 antiprotons each. To avoid spatial blow-up of the cloud due to the internal energy spread the particles will be accelerated to approximately $1 \mathrm{keV}$ to minimize the transfer time. 
They will then be decelerated at the entrance to the second trap and dynamically captured. In the current configuration the radial extend of the cloud in the catching trap is only defined by the spot size of the antiproton beam hitting the final degrader foil and is therefore only limited by the $5 \mathrm{~mm}$ active diameter of the trap entrance. Particles being ejected from the trap will follow the magnetic field lines and a $5 \mathrm{~mm}$ diameter cloud (or uncertainty in definition of the center of the cloud) will expand to about $20 \mathrm{~mm}$ diameter by the time the particles reach the fringe magnetic field at the end of the cryogenic bore. At this location electrostatic einzel lenses are introduced to refocus the beam. Additionally, the antiproton cloud will subsequently be re-compressed upon entering the 2 $\mathrm{T}$ magnetic field of the gravity experiment.

While a fast transfer of a single pulse is required for the gravity experiment, most other experiments with ultra-low energy antiprotons will require a 'semi'-continuous beam, possibly with timing information on the release of individual antiprotons. A number of possible schemes can be envisioned to extract the cloud of antiprotons from the PS200 catching trap in such a way.

One can not just lower the potential at one end cap over an extended period of time. Since all antiprotons will have been cooled to an extreme low temperature one would only obtain an extraction during the very last fraction of the spill time. Instead, one can eject the antiprotons by an evaporative process. Here the axial or cyclotron resonance frequency of the stored antiprotons is weakly excited, leading to a continuous heating and a slow 'boil-off' of particles from the well. The rate of boil-off can be controlled by the amplitude of the radio-frequency applied, as well as by the detuning between the applied frequency and the resonant frequency. Test experiments conducted at Los Alamos using a smaller Penning trap filled with protons have generated continuous spills of protons for approximately 30 minutes at a time [19].

This evaporative slow spill can be used for experiments where a low intensity of antiprotons and no timing information is needed. If a time structure is required (i.e. for time-of-flight studies of the energy loss in materials) a different method is proposed. This method was originally developed to eject low energy electrons from a Penning trap and to study their time-of-flight through an inhomogeneous magnetic field to measure the electron magnetic moment [20]. As described before, the well depth is slowly reduced, allowing electrons to leave the trap whenever their kinetic energy exceeds the well depth. Superimposed onto this linear ramp is a series of triangular spikes with a half width longer than the oscillation period of the particles in the harmonic well. During the time period of 
one of these pulses all electrons occupying the energy band covered by the pulse amplitude are allowed to escape, generating a micro bunch with a defined start time.

A derivative of this method has been used during our test experiments: A sequence of triangular pulses with a FWHM slightly larger than the oscillation period of the trapped particles, and an amplitude of 1 - 2 Volts was superimposed onto the constant trapping voltage. Additionally, a weak RF drive was applied at the axial resonance (or the cyclotron resonance) to continuously heat the particle cloud. The amplitude of this drive was established in such a way, that continuous boil-off was not quite taking place yet. A multi channel analyzer with a $200 \mathrm{~ns} /$ channel time resolution was triggered with the leading edge of each of these pulses and a time spectrum for 1000 individual pulses was obtained in this fashion. Two examples are displayed in figure 6 . In case (a) the RF drive is set appropriately and we observe a well defined peak in the time window between $3.0 \mu \mathrm{sec}$ and $4.2 \mu \mathrm{sec}$, with very little background afterwards. This time window is correlated to the length of the exit electrode, which the particles are traversing at their lowest energy, and the amplitude of the release pulse, i.e. the energy spread of the released particle bunch. In case (b) the RF drive was chosen slightly higher, and an elevated background of randomly escaping particles stemming from over-heating was observed. In some tests it was found that the RF heating could be omitted if the Fourier spectrum of the pulse contains a sufficient component at the particles' resonance. The spacing between the pulses can be chosen arbitrarily, allowing the generation of a 'pseudo' beam of antiprotons, consisting of many micro pulses with a few particles in each pulse (in figure $6 \mathrm{~b}$ less than one on average) at an arbitrary repetition rate.

\section{Possibilities of ultra-low energy antiproton experiments.}

The availability of low energy antiprotons with a well defined energy has generated substantial interest amongst experimenters who have studied low energy antiproton phenomena over the last years. While I refer the reader to the extensive literature on these experiments, I would like to use two specific examples to discuss the current methods used, and to present an alternative method, and its advantages, based on our catching trap.

The first group of experiments is a series of measurements on energy loss and straggling of antiprotons passing through matter $[21,22]$. These experiments were performed by passing the lowest energy beam available from LEAR ( $5.9 \mathrm{MeV})$ through a degrader material and then using a time-of-flight tag to select the particles with a specific energy. This method has distinct disadvantages at lower energies. If the thickness of the 
degrader material is increased to reduce the beam energy below approximately $1 \mathrm{MeV}$ both the energy spread and the angular spread increase dramatically. As shown in figures 3.9 and 3.10 of reference 21 , the energy spread becomes equal to the mean energy at approximately $1-2 \mathrm{MeV}$, and the number of particles available at a given energy decreases drastically below $1 \mathrm{MeV}$. Under these conditions one can no longer speak of a 'beam of antiprotons'. Not only is the number of antiprotons available at the energy of interest diminishing rapidly, requiring more and more integrated beam time from the antiproton source to accumulate appropriate statistics, but also does the background due to 'unwanted' particles at higher energy become quickly overwhelming. These high energy particles may annihilate in the experimental set-up, producing false counts, and even saturating the detector system.

Here our catching trap would serve as a bunching system to compress the phase space occupied by the antiprotons and to remove the high energy background from the measurement. By utilizing a $30 \mathrm{kV}$ well depth and by cooling the particles to less than 1 $\mathrm{eV}$ one achieves an enhancement of more than $10^{4}$ in energy density. This will allow experiments to explore energy regimes far below the current limit of 10 's of $\mathrm{keV}$ and to accumulate a much better statistics in the low energy region than possible so far. The OBELIX collaboration has presented measurements of $\mathrm{dE} / \mathrm{dx}$ in gaseous targets to energies as low as $3 \mathrm{keV}$ using a combination of time-of-flight and spatial vortex tagging [23]. The analyses presented by that group strongly relies on proper deconvolution of their integral data over a wide energy range and it has been shown that the results obtained may not be entirely conclusive. Using the PS200 catching trap, a well defined energy beam can be produced with a very small energy spread, allowing the direct measurement of low energy processes. A number of such experiments have been proposed by the PS194 collaboration [24] and, once approval has been obtained, could be performed within the next years with a much reduced impact on the LEAR operation.

A second group of experiments which would greatly benefit from very low energy, narrow energy width, antiproton beams are those requiring ultra-thin targets. One example is the study of the formation and delayed decay of hypernuclei when antiprotons are stopped in thin target foils. These processes were studied at LEAR by the PS177 collaboration [25]. A shadowing method was used to distinguish between prompt decays inside the target and delayed decays of hyper nuclei which had escaped the target. The lifetime of heavy hyper nuclei in the region of uranium was measured to be of the order of $10^{-10}$ seconds. To improve this method it would be desirable to use thinner targets, thus 
allowing a larger fraction of the formed hypernuclei to escape. To maintain a reasonable stopping rate in these ultra-thin targets a much lower energy of the antiprotons would be required.

These again could be obtained from our catching trap. By using the time structured extraction method described in the previous section one can generate short micro bunches of antiprotons at energies from a few hundred electronvolts up to 30 kilo-electronvolts by switching the potential of the long, cylindrical, high voltage exit electrode (or another electrode placed in the system for this specific purpose) during the time the bunch is shielded from external potentials while inside this electrode. The bunches will then be accelerated to the kinetic energy set by the potential applied to this electrode upon exiting. Model calculations indicate a near 100\% efficiency for this process and experimental tests to characterize this beam structure are under way [19]. As an additional benefit the overall intensity of antiprotons entering the experimental set-up is greatly reduced and the background from annihilations outside the target can be made to be near zero.

We currently study the possibility to measure possible signatures for neutron and proton "halos" through prompt X-ray, Gamma-ray, and annihilation particle emission. Low energy antiprotons entering a nucleus will preferably annihilate near the nuclear surface. In these cases the resulting pions have a high probability to miss the rest of the nucleus, thereby avoiding excessive excitation of the nucleus and subsequent fission of the target, and the result is a "cold" daughter product with proton and neutron numbers of either $(Z-1, N)$ or $(Z, N-1)$, depending on whether the annihilation occurred on a neutron or proton. By detecting a distinct signature for the two possible routes the neutron distribution near the nuclear surface can be mapped out. J. Jastrzebki et al. [26] have presented the first measurements based on these ideas, but their experimental method was limited to such cases, where both daughters were radioactive, since off-line radiochemistry methods were used for the reaction analysis. Our experiment will not be limited to radioactive annihilation products. Ultra-thin isotopically enriched targets and prompt measurements will be used to take advantage of the low energy properties of the extracted antiproton beam from the PS200 catching trap. Rather than studying the subsequent radioactive decay of the daughter products we plan to observe the (prompt) de-excitation of the daughters from the excited state to the ground state in situ. As an example a case which is also of theoretical interest [27] is described here. Figure 7 shows a partial level diagram for the reactions ${ }_{20}^{48} \mathrm{Ca}_{28}+\overline{\mathrm{p}}(\mathrm{p}) \rightarrow{ }_{19}^{47} \mathrm{~K}_{28} \rightarrow{ }_{20}^{47} \mathrm{Ca}_{27}, \quad$ and ${ }_{20}^{48} \mathrm{Ca}_{28}+\overline{\mathrm{p}}(\mathrm{n}) \rightarrow{ }_{20}^{47} \mathrm{Ca}_{27} \rightarrow{ }_{21}^{47} \mathrm{Sc}_{26}$. Shown are the different prompt and delayed decay 
signatures, which can be used for the identification of the reaction branch. In both cases

the final product will decay into ${ }_{22}^{47} \mathrm{Ti}_{25}$ which may be used for calibration of the total reaction rate. In addition to yielding significant new physics results, this experiment will be useful in characterizing the extracted beam quality for the transfer to the PS200 gravity experiment.

Another experiment that has been discussed proposes to use extremely thin gas targets to study the initial state distribution of antiprotonic atoms [24, appendix 7]. Using extreme low energy antiprotons $(10-1000 \mathrm{eV})$ one can use a dilute gas target $(1 \mathrm{~cm}$ length at $10^{-3}$ Torr) and still maintain a reasonable high reaction rate (10/second). Both auger electrons and the recoiling antiprotonic atoms can escape the thin target and can be used to study the physics of the capture process.

\section{Antihydrogen production.}

The simplest system which can be studied by atomic physicists, the only one where theory can even attempt to find exact solutions, and the one which has subsequently been studied with great precision and success, both theoretically and experimentally, is the hydrogen atom [28]. Naturally, it is a tantalizing thought to be eventually able to study with the same precision its mirror image, the antihydrogen atom, either to solidify, or to expand our understanding of fundamental symmetries.

By stating this goal, I restrict myself to the discussion of possibilities to obtain antihydrogen in an experimental environment suitable for precision measurements comparable to those achieved on the hydrogen atom. Adding the novel restriction that antihydrogen will always be an extremely rare object, one immediately realizes that it is a sensible approach to cool and trap the antihydrogen atoms.

Much of the work which will be described in this section has been performed by other groups. I only take the liberty to summarize and comment on a variety of issues, and hope that this will initiate a deeper discussion amongst groups interested in the future of this field. For a more detailed technical view, the reader is advised to consult the references given throughout this section. 


\subsection{The different schemes for antihydrogen production.}

A variety of schemes for producing antihydrogen has been proposed, and discussed in some detail [29-42], with the first mentioning of the possible production of antihydrogen in traps by Dehmelt and co-workers [43]. Below I list a selection of these processes (with appropriate references), of which only a few will be discussed here in detail. The first line shows the basic production mechanism of combining a positron and an antiproton to form a neutral atom. A photon in the final state carries away excess energy and momentum. All following schemes (except the last one) are based on attempts to increase the effectiveness of recombining antiprotons and positrons by either laser stimulation of the radiative recombination process [31], or by using a three-body process [32-40]. The last line describes a method which is currently pursued at Fermi Lab [41] and at CERN [42], where an antiproton picks up a positron from Bremsstrahlung pairs generated when the antiproton passes through a gas target. The resulting antihydrogen atom will have an extraordinary high energy (at least $5.9 \mathrm{Mev}$ at LEAR, several GeV at Fermilab), which will, despite the fact that the authors of reference 41 propose to perform a $1 \%$ measurement of the Lambshift, render it useless for high precision measurements. But this method may have already yielded the first observation of antihydrogen atoms produced in the laboratory [44].

$$
\begin{aligned}
& \begin{aligned}
\mathrm{e}^{+}+\overline{\mathrm{p}} \Longrightarrow \mathrm{H} & =\mathrm{H} v \\
\mathrm{nh} v+\mathrm{e} & =\mathrm{\textrm {H }}+\mathrm{h} v+\mathrm{nh} v
\end{aligned} \\
& \mathrm{e}^{+}+\mathrm{e}^{+}+\overline{\mathrm{p}}=\overrightarrow{\mathrm{H}}+\mathrm{e}^{+} \\
& \mathrm{e}^{-}+\mathrm{e}^{+}+\overline{\mathrm{p}} \Rightarrow \overline{\mathrm{H}} .+\mathrm{e}^{-} \\
& \mathrm{e}^{+}+\overline{\mathrm{p}}+\overline{\mathrm{p}}==\Rightarrow \overline{\mathrm{H}}+\overline{\mathrm{p}} \\
& \text { Ps }+\overline{\mathrm{p}}====\overline{\mathrm{H}}+\mathrm{e}^{-} \\
& \mathrm{Ps}^{*}+\overline{\mathrm{p}}==\Longrightarrow \overline{\mathrm{H}}^{*}+\mathrm{e}^{-} \\
& \overline{\mathrm{p}}+\mathrm{Z} \Rightarrow \overline{\mathrm{p}}+\mathrm{e}^{+}+\mathrm{e}^{-} \stackrel{=}{=} \overline{\mathrm{H}}+\mathrm{e}^{-}
\end{aligned}
$$

For all practical purposes, only processes (3), (6), and (7) deserve close attention by the trap community. In the first case, both constituents forming antihydrogen need to be trapped, while in the last two cases only antiprotons need be controlled before the recombination process, while the positron is delivered in form of a beam of positronium. Both methods have distinct advantages and disadvantages and, dependent on the final application, either one might be the better choice. 


\subsubsection{Antihydrogen production using trapped plasmas.}

This method has originally been proposed, and discussed extensively, by G. Gabrielse's group [33]. In a "nested trap" scheme, forming two Penning traps, the oppositly charged constituents for antihydrogen production are held in separate clouds and cooled to $4 \mathrm{~K}$, or possibly below [45]. At a definite time, the two clouds are merged by lowering the electrostatic barrier between them, and antihydrogen is formed. The rate constant for this process is strongly temperature dependent :

$$
\Gamma=6 \times 10^{-12}(4.2 / \mathrm{T})^{9 / 2} \mathrm{n}_{\mathrm{e}}^{2} \mathrm{~s}^{-1}
$$

and therefore benefits vastly from cooling the particles. Because of the mass difference between positrons and antiprotons, with positrons at $4.2 \mathrm{~K}$, the antiprotons could have energies as high as $1 \mathrm{eV}$ before the recombination rate is significantly affected. As Gabrielse points out [33], this could be used to form $\mathrm{eV}$ energy beams of antihydrogen atoms leaving the trap volume. The beam quality would strongly depend on how well the axial energy of the antiprotons could be decoupled from the radial energy.

While the rate can be extremely high (with $10 \% \mathrm{~cm}^{3}$ positron density at $4.2 \mathrm{~K}$ one obtains $\Gamma=6 \times 10^{6} / \mathrm{s}$ ), one specific problem must be addressed: The antihydrogen atoms are being formed in highly excited Rydberg states $(n>100)$, and need to be quickly deexcited, before electrostatic field gradients from the Penning trap will ionize these states, or before spectroscopic studies could be attempted. This de-excitation process also effects the capability to trap the antihydrogen atom once formed (see section 4.3 ).

Alternative methods to store both positrons and antiprotons simultaneously have been proposed. A Paul trap, which is insensitive to the sign of the charge could be used for simultaneous confinement in the same volume [43]. Also, the potential use of a combined Paul and Penning trap for simultaneous confinement of positrons and antiprotons for the purpose of antihydrogen formation has been pointed out [46]. On the other hand, keeping the positrons and antiprotons apart until they are cooled to extremely low temperature energy, and then producing antihydrogen with high rate constants as a well defined burst may have advantages for a number of applications. 


\subsubsection{Antihydrogen production by positronium-antiproton collisions}

Alternatively to the method described above, one may enhance the radiative antihydrogen formation rate by several orders of magnitude through coupling of the recombination process to a third particle for energy and momentum conservation. Based on this idea is a proposal to utilize collisions between positronium atoms and antiprotons [38]. This process can be interpreted as Auger capture of the positron to the antiproton and cross sections have been estimated by Humberston et al. [37], using charge conjugation and time reversal to link the cross section for positronium formation in collisions between positrons and hydrogen to the antihydrogen formation cross sections. Early calculations assumed both $\overline{\mathrm{H}}$ and Ps to be in the ground state and obtained a broad maximum of the cross section of $3.2 \times 10^{-16} \mathrm{~cm}^{2}$ at a kinetic energy of approximately 2.5 $\mathrm{KeV}$ of the antiprotons. Calculations of the total $\overline{\mathrm{H}}$-formation cross section using classical and semi classical methods [47] have obtained values of $\sigma_{\overline{\mathrm{H}}}$ which are considerably larger than the ground-state results. Values for the formation of $\overline{\mathrm{H}}$ in excited states are given by Ermolaev et al. [48] and indicate that there is a large cross section to low-lying excited $\overline{\mathrm{H}}^{*}$ states, which therefore would be directly accessible to spectroscopic studies.

M. Charlton [40] has discussed the formation of excited $\overline{\mathrm{H}}^{*}$ atoms via collisions between antiprotons and excited positronium. The cross section follows a classical $n_{P_{s}}^{4}$ scaling, where $n_{P_{s}}$ denotes the principal quantum number of the positronium atom, leading to large enhancements in the reaction rate. This process can also be utilized to preferentially populate specific low level excited states for spectroscopic purposes. An additional feature of the excited state process is the reduced recoil energy of the resulting $\overline{\mathrm{H}}$ atoms. By energy and momentum conservation the-recoil temperature arising from the binding energy difference $\Delta \mathrm{E}$ between $\overline{\mathrm{H}}$ and $\mathrm{Ps}^{*}$ is given as

$$
\mathrm{T}_{\mathrm{H}^{*}}^{-}=2 \Delta \mathrm{Em} / 3 \mathrm{kM}=28.6\left(\frac{2}{\mathrm{n}_{\overline{\mathrm{H}}}^{\frac{2}{2}}}-\frac{1}{\mathrm{n}_{\mathrm{Ps}}^{2}}\right) K,
$$

where $m$ and $M$ are the mass, and $n_{\bar{H}}$ and $n_{P_{s}}$ are the principal quantum numbers of the positron and antiproton, respectively. Waiving the requirement of negligible kinetic energy of the positronium would add additional terms of the order $\mathrm{mT}_{\mathrm{Ps}_{\mathrm{s}}}{ }^{*} / \mathrm{M}$. Resonant charge transfer is taking place for $n_{\bar{H}}=\sqrt{2} n_{p_{s}}$, when the initial and final states are degenerate in energy. A range of $\bar{H}^{*}$ states close to resonance will be preferentially populated, allowing a certain degree of selection of the final $\overline{\mathrm{H}}^{*}$ excited state. 
A final note on rates: much discussion has been put forward about the difference in rate constants for $\overline{\mathrm{H}}$ production using either one of the processes discussed here. In essence, this discussion may be viewed as non relevant if the product $\bar{H}$ is to be trapped. When only a limited number of antiprotons is available to work with, there is no fundamental difference between a rate of $10^{6} / \mathrm{sec}$, converting all antiprotons to $\overline{\mathrm{H}}$ in a single second, or a rate of $1000 / \mathrm{sec}$ (or even $100 / \mathrm{sec}$ ), requiring a production cycle of 16 minutes (or 3 hours), which still is much shorter than the storage time of antiprotons in traps of several months. The antihydrogen atoms produced are intrinsically stable and long term storage must be a goal for experimental reasons anyway.

\subsection{Trapping of positrons}

\subsubsection{Production and trapping of low energy positrons}

Since the rate constant for recombination of antiprotons and positrons in plasmas is proportional to the square of the positron density, it is important to accumulate significant numbers of positrons and compress them into a small volume. Densities typically considered by the authors of these proposals are $10^{7} \mathrm{e}^{+} / \mathrm{cm}^{3}$. A number of schemes are under investigation for achieving these densities in traps. The very first capture of positrons into a Penning trap dates back to 1980. Dehmelt and co-workers [4] used a radioactive source $\left(0.5 \mathrm{mCi}\right.$ of $\left.{ }^{22} \mathrm{Na}\right)$ placed off axis outside the end cap of a Penning trap. From those positrons emitted by the source which enter the trap, the minute fraction with sufficiently low axial kinetic energy were deflected by the opposite endcap. By the time they returned to the entrance hole they had undergone a small azimuthal drift due to the magnetron motion ( $\mathrm{E} \times \mathrm{B}$ drift) and saw a slightly higher potential. This enhanced the time period before the positrons could possibly escape from the trap by a factor $\omega_{z} / \omega_{m}(\cong 2000)$. During this time, enough axial energy could be extracted by coupling the axial motion to an external damping resistor so the positrons could be trapped permanently. The total efficiency of this method was only 3 out of $10^{10}$ captured positrons ( $23 \mathrm{e}^{+}$/hour), quite sufficient for the experiment at the time, but somewhat too low for the purpose of reaching the high densities required for antihydrogen production.

An important first step to improve this situation is the reduction of the energy of positrons emitted from radioactive sources, or produced at linear accelerators, using moderators. Positrons from radioactive sources and from LINAC production targets exhibit a broad continuous spectrum with very high upper energy values. If these positrons are implanted into a solid, most are thermalized 0.1 to $1 \mathrm{~mm}$ deep from the surface, and a 
substantial fraction will diffuse back to the surface of the material. These positrons are the ones which can, if sample and surface conditions are suitable, be re-emitted from the surface before annihilation. Some materials show a negative work function for positrons, therefore emitting positrons as a low energy $(\sim \mathrm{eV})$, mono-energetic $(\Delta \mathrm{E} \sim \mathrm{l} \mathrm{eV})$ beam, preferably normal to the surface. A wealth of references exists in the literature on this topic, with recent reviews by Schultz and Lynn [49] and M. Charlton and G. Laricchia $[50]$.

The challenge remains to trap these positrons in a trap, where they can then be allowed to recombine with the antiprotons by either merging the clouds or by allowing the antiprotons to traverse the positron cloud with $\mathrm{eV}$ type kinetic energies. C. Surko et al. [51] trapped of the order of $10^{7}$ positrons for several minutes using collisions with a buffer gas for cooling. This environment is incompatible with the extreme vacuum requirements for antiproton storage, but one can easily envision to develop a final UHV stage for this experiment, in which positrons could be accumulated and stored before being transferred to the antiproton trap. Since positrons can be very efficiently remoderated by using thin foils, positrons can be accelerated to several $\mathrm{keV}$, and then passed through a second foil separating the ultra-high vacuum (UHV) volume of the positron accumulator from the static cryogenic vacuum (SCV) of the antiproton trap.

A scheme to stack and accumulate positrons has also been proposed by $\mathrm{R}$. Conti et al. [52] using a dynamic two trap system. The first confining potential is increased continuously at a rate sufficiently high, that positrons entering the potential well from a moderator can not reach the moderator again on the first bounce. Once a specified potential is reached $(\sim 5 \mathrm{kV})$, the accumulated positrons are accelerated towards a second moderator and injected in a similar fashion into the final storage volume [36.52]. $\cdots$

The last method discussed here has been proposed by Brown et al. [53] and recently has been demonstrated [54]. Using the same basic scheme as Dehmelt et al. [4] it relies primarily on the higher intensity of low energy positrons available through above described moderator techniques. Using a $10 \mathrm{mCi}{ }^{22} \mathrm{Na}$ source and a $\mathrm{W}^{110}$ moderator in reflection geometry a loading rate of $0.2 \mathrm{e}^{+} / \mathrm{sec}$ and a maximum total number of positrons of $3.510^{4}$ has been achieved, representing an improvement in efficiency by a factor of 1.4 over the previous experiments. The main difficulty in this experiment was posed by the magnetic field gradient between source and moderator, resulting in a significant loss of primary positrons. Subsequently to the trapping the positron cloud is compressed by 
sideband centering of the magnetron motion to achieve the proposed positron density of $10^{8} \mathrm{e}^{+} / \mathrm{cm}^{3}$.

\subsubsection{Sources of positronium.}

For reactions (6) and (7) positronium in its ground state or in excited states respectively is required. Ground state positronium exists in two states, a singlet and a triplet configuration. While the singlet state has a prohibiting short life time ( $150 \mathrm{psec}$ ), the positronium life time in the triplet state is $142 \mathrm{~ns}$, sufficiently long for the positronium atom to travel several centimeters at the $\mathrm{eV}$ energies it is formed at before it annihilates into three photons. In the presence of a magnetic field some of the substates are quenched out by admixture of a singlet component, but $2 / 3$ remain in a pure state, maintaining their long life time. For excited positronium not only the cross section for antihydrogen formation, but also the life time scales like $\mathrm{n}_{\mathrm{Ps}}^{4}$, possibly an important feature for the benefit of the experiment.

In many materials a non-negligible probability exists that positronium (Ps) will form as a positron leaves the solid surface and travels through the decreasing electron density just outside. Conservation of energy gives a positronium formation potential, which in many cases can be negative, assuring the positronium formation to be one of the main branches for positrons diffusing back to the surface. First efficient positronium formation was observed by Canter et al. [55]. Mills later showed the effect to be $30 \%$ to $100 \%$ on well characterized surfaces, and also studied the dependence on the incident positron energy [56].

Excited positronium with $\mathrm{n}_{\mathrm{Ps}}$ ranging from 13 to 19 has been produced by Ziock et al. [57], using a two quantum excitation. The production of highly excited Ps by recombination of merged $\mathrm{e}^{+}$and $\mathrm{e}^{-}$plasmas has also been observed by Cowan et al. [58]. Still, significant work lies ahead in adapting all these techniques into a single experiment.

\subsection{Trapping of antihydrogen.}

As stated several times before, the ultimate goal of a sensible experimental program must be to confine the formed antihydrogen in space. Only then can one achieve an appreciable precision in the spectroscopic measurements with the very limited amount of antihydrogen available by any method. Much of the development work on trapping antihydrogen atoms can be guided by the excellent work by the groups at MIT $[59,60,61]$ 
and Amsterdam [62], who have developed technologies to magnetically confine neutral hydrogen atoms.

The confining force being the magnetic gradient interaction with the magnetic moment separates the hydrogen in the high-field seeking $(\mathrm{H} \uparrow)$ and the low-field seeking component $(\mathrm{H} \downarrow:(\mathrm{F}=1, \mathrm{~m}=1$ and $\mathrm{F}=1, \mathrm{~m}=0))$. Even though work with hydrogen has been performed with both states, only the low field seeking states are of use in the case of antihydrogen, where collisions with the walls of the containment vessel are clearly unacceptable. The trap configuration used for the latter case normally consists of an arrangement of coils, designed to produce a magnetic minimum at the center of the trap without having a zero field location, which would introduce spin-depolarizing Majorana transitions. The essentially cylindrical geometry of these traps has been proposed by Pritchard et al. [57] and provides transverse trapping forces by a set of super conducting race track coils which generate a quadrupole field. Axial confinement is achieved through coaxial solenoids at either end of the trapping volume, which provide a barrier against axial leakage and also the non-zero field value in the center. As a specific example, the magnetic field values in the Amsterdam trap [62] are 1.5 Tesla for the quadrupole fields at $r=6.5 \mathrm{~mm}$ and 1.5 and 1.7 Tesla for the coaxial coils respectively, with the field minimum at the center adjustable between $\mathrm{B}=0$ and $\mathrm{B}=1 \mathrm{~T}$.

In filling the magnetic traps in the hydrogen work, the "gas" is allowed to fall into the potential well by inelastic collisions with residual gas atoms, a method unacceptable for antihydrogen. A possible alternative would consist of either superimposing the magnetic trap and the Penning trap in a way where the Penning trap is located within the potential well for the confinement of the neutral atoms. Antihydrogen produced in the high-field seeking states would quickly leave the trap volume, while the $(\overline{\mathrm{H}} \uparrow)$ states would be attracted towards the center. To achieve continuous confinement, the well depth will have to be substantially larger than the kinetic energy at which the antihydrogen is formed, or effective laser cooling needs to be employed, to quickly cool down the antihydrogen atoms after formation. Using 1.5 Tesla for the magnetic well depth, initial confinement would be limited to atoms with $T<1$ Kelvin. Active laser cooling is needed to increase this value as well as to cool the antihydrogen atoms to the center of the well for long term confinement. For this purpose it is not entirely necessary to have a CW Lyman $\alpha$ cooling laser available. A pulsed laser would be sufficient when one starts with such low energy atoms since it is only necessary to absorb approximately 10 photons (under maximum momentum transfer conditions) to essentially stop the antihydrogen atoms. 
A serious concern must be raised with respect to the spin polarization requirement to initially stabilize the antihydrogen atoms. Only atoms with electron spin $+1 / 2$ will be low field seeking, and therefore confined by the magnetic configuration. If antihydrogen atoms would be formed only in the ground state the statistical population of these states would be high enough from the beginning. But what if the antihydrogen is formed in low lying excited states, or even in high $\mathrm{n}$ Rydberg states? The routes of de-excitation will obviously proceed via all possible magnetic sublevels, many of them not trapable. The radiative lifetime in highly excited states is sufficiently long for the antihydrogen atom to drift to the edge of the trap and annihilate before the next spontaneous transition would possibly bring it back to a trapable state. Starting at $n=100$ and assuming complete statistical balance between all substates and radiative decay only, this would lead to a reduction of the number of antihydrogen atoms trapped at the end of 30 orders of magnitude! To reduce the time spent in highly excited states one can partially rely on collisional de-excitation, provided the antihydrogen atoms do not drift outside the positron plasma within the time scale of typical collision times. The better method would involve a laser tuned to the energy difference between the band of preferably populated Rydberg states and a specific excited state from which then spectroscopic measurements could proceed.

\section{Precision measurements using antihydrogen.}

Considering the effort necessary to produce antihydrogen one must naturally ask the question what the physics benefits of such an endeavor would be. In principle, these can be found in two areas: Comparison of results of spectroscopic measurements of hydrogen and antihydrogen which would constitute a test of CPT at a level rivaling even the result on the kaon system and the study of the gravitational interaction of antimatter with the Earth's gravitational field, testing the validity of the weak equivalence principle (WEP) and possibly shedding light onto the problem of unifying gravity with the three other forces.

\subsection{CPT tests and precision spectroscopy}

The precision in spectroscopic studies of hydrogen advanced enormously over the last decade. Today the highest precision has been achieved for the Hyperfine Structure $\left(6.4 \times 10^{-13}\right)$ and for the $2 \mathrm{~s}-2 \mathrm{p}$ Lambshift $\left(1.8 \times 10^{-11}\right)$, and based on the lifetime of the $2 \mathrm{~s}$ state of $1 / 8$ second and the natural linewidth connected to this a precision of $10^{-18}$ has been speculated. This latter precision will also most likely require using trapped hydrogen 
atoms, an environment which would be directly applicable to antihydrogen. Currently the best tests of CPT invariance have been performed in the kaon system followed by precision comparisons of magnetic moments and masses of electron, positron, proton, and antiproton. The comparison of the inertial masses of proton and antiprotons have now reached a precision of $1.4 \times 10^{-9}$ [63]. In the strict sense this must be considered only a measurement of the ratio of the charge-to-mass ratios of the two particles. It has been proposed [64] to combine the direct determination of the cyclotron frequencies with the measurement of the Rydberg of protonium to extract a independent CPT test, but with the current precision on this quantity [65] a CPT test of only $2 \times 10^{-5}$ is possible. Using the Rydberg of Antihydrogen, one can construct a limit for the charge equality between antiproton and proton which is entirely based on frequency measurements, and could therefore yield a direct test of CPT at a level of $10^{-11}$.

\subsection{Antimatter Gravity.}

Often the argument is made, that measuring gravity on charged antimatter is nearly impossible due to the interaction of the charge with stray electric fields, and that it would be advantageous to use a neutral particle instead. To compare these two methods in a fair way one needs not only consider the added complication of producing the antihydrogen atoms but must also device a possible method of measuring the gravitational acceleration with sufficient precision.

Even though it is repeatedly being mentioned, purely ballistic methods to measure gravitational acceleration on antihydrogen atoms may be ruled out. Even if the antihydrogen atoms can be laser cooled to the photon recoil limit of $T=\frac{1}{2} \Gamma \hbar / k=2.4$ $\mathrm{mK}$, this temperature would still correspond to a distribution of height of approximately 1 $\mathrm{m}$ in the gravitational field. A precise determination of the centroid of a cloud of this dimension is not possible. Similarly, if one should be able to generate an atomic fountain with a mean energy of the photon recoil and a spread of half that value, the observed time-of-flight over a height of $10 \mathrm{~cm}$ will be $14 \mathrm{msec} \pm 7 \mathrm{msec}$, again not yielding a precision measurement of $g$. The only hope in the latter case would be to perform an end point measurement similar to the PS200 proposal, but this time with more particles near the end point.

A potentially much more powerful method could be developed based on the work of by S. Chu and collaborators [66]. In their experiment they have used velocity sensitive stimulated Raman transitions to measure the gravitational acceleration, $\mathrm{g}$, of laser cooled 
sodium atoms in an atomic fountain geometry. An ultra cold beam from an atomic trap is launched upwards and is subjected to three subsequent pulses to drive a two-photon Raman transition between the $F=1$ and 2 levels in the $3 S_{1 / 2}$ state. A Raman transition is used to provide a large photon recoil velocity while satisfying the metastability of the states necessary for long interaction times. A first $(\pi / 2)$ pulse will prepare the sample in a superposition of the $\mid 1, \mathbf{p}>$ and the $\mid 2, \mathbf{p}+\bar{k} \mathbf{k}>$ states; the second $(\pi)$ pulse will reverse the populations, and the third $(\pi / 2)$ pulse will bring the wave packets to interference. The interference can be detected by probing the number of atoms in state $|2\rangle$. In the absence of any external forces acting on the atoms the final state of an atom will depend on the phase of the driving Raman field. This result can be extended to an atom falling freely in the gravitational field. In the frame of reference falling with the atom the Raman light fields appear Doppler shifted linearly in time, which shows up as a phase shift varying as the square of the time: $\Delta \phi=-\left(\mathbf{k}_{1}-\mathrm{k}_{2}\right) \mathrm{gT}^{2}$. Since the Doppler shifts $\approx 2 \mathrm{k}_{1} \mathrm{gT}$ are much larger than the Rabi frequency, an active frequency shift between the three subsequent pulses was used to compensate for the deceleration of the atoms in the fountain.

Using a $50 \mathrm{~ms}$ delay in-between the pulses, distinct interference fringes were observed, and a least square fit to the data gave an uncertainty in the phase determination of $3 \times 10^{-3}$ cycles. This represented a sensitivity to $\mathrm{g}$ of $\Delta \mathrm{g} / \mathrm{g}=3 \times 10^{-8}$. A higher sensitivity is expected to be obtainable when cesium is used instead of sodium because of large reduction of the $\mathrm{rms}$ velocity spread. The current work was done with $30 \mu \mathrm{K}$ sodium atoms (representing a $30 \mathrm{~cm} / \mathrm{s}$ velocity spread). For the cesium sample one may expect an $\mathrm{rms}$ spread of $2 \mathrm{~cm} / \mathrm{s}$ only, therefore a much larger portion of the sample will be contributing to the fringes.

Hydrogen is (and antihydrogen certainly would be even more) ill suited for high precision measurements, notwithstanding the enormous advances in hydrogen spectroscopy over the last years [67], and a translation of this method to the hydrogen/antihydrogen case will not be trivial and straight forward. A large problem will be imposed by the much higher photon recoil limit for laser cooling hydrogen atoms ( $\approx 3$ $\mathrm{mK}$ ) which gives an $\mathrm{rms}$ velocity spread of approximately $700 \mathrm{~cm} / \mathrm{sec}$. A much faster fountain beam, resulting in greatly increased experimental dimensions, will have to be used. Therefore a much larger fraction of the initial beam pulse will be lost due to ballistic spreading during the flight time of the sample and much less than $1 \%$ of the initial population can be expected to contribute to the fringes. This will also cause severe problems for the antihydrogen/hydrogen comparison, since the supply of antihydrogen 
atoms will be limited to small numbers and a re-trapping scheme needs to be incorporated into the experiment. Nevertheless, this method is the only one identifiable in the current literature which shows the potential of a high precision measurement of $g$ on antihydrogen atoms.

\section{Summary}

Recent advances in trapping and cooling of antiprotons have opened up new opportunities for research with ultra-low energy antiprotons. Already now significant improvements in testing CPT in the baryon sector have been accomplished, but the ultimate precision will only be possible once antihydrogen can be formed and contained. Long storage times of antiprotons and protons as well as recent advances in trapping and cooling of neutral hydrogen bring this once futuristic idea into the realm of the technical possible, even though experimentally challenging. A number of crucial steps still need to be proven, but at this time the largest challenge seems to be the continuation of a supply of antiprotons at low enough energy to continue the line of work presented here. The possibility of extreme high precision spectroscopy on antihydrogen and the wide spectrum of interesting physics covering atomic, nuclear, and gravitational physics should well be sufficient to justify such a modestly expensive facility and the interested community needs to actively pursue these ideas.

\section{Acknowledgments.}

The support of all members of the PS200 collaboration in much of the work presented here is gratefully acknowledged. Current members include R. E. Brown, T. W. Darling, P. L. Dyer, T. Goldman, D. Hajdukovic, M. H. Holzscheiter, K. Hosea ; R. A: Kenefick, N. S. P. King, R. A. Lewis, G. L. Morgan, M. M. Nieto, T. Otto, S. P. Parry, R. Ristinen, J. Rochet, M. M. Schauer, G. A. Smith, and F. C. Witteborn. On the issues of antihydrogen production I enjoyed numerous enlightening discussions with B. Deutch, $\mathrm{G}$. Laricchia, M. Charlton, and all members of the UCL - Aarhus - Los Alamos antihydrogen group. This work was supported by the US Department of Energy under contract no. W7405 ENG-36. 


\section{REFERENCES:}

1. N. Beverini et al., CERN Proposal PSCC/86-2/P94;

2. G. Gabrielse, X. Fei, L. A.Orozco, R. L. Tjoelker, J. Haas,

H. Kalinowsky, T. A. Trainor, W. Kells; Phys. Rev. Lett. 63 (1989) 1360

3. M. H. Holzscheiter et al.; Nucl. Phys. A558 (1993) 709c

4. P. B. Schwinberg, R. S. Van Dyck Jr., and H. G. Dehmelt; Phys. Lett. 81A, 119 (1981)

5. C. M. Surko, M. Leventhal, and A. Passner; Phys. Rev. Lett. 62, 901 (1989)

6. P. Lefevre; in "Physics at LEAR with Low Energy Antiprotons"; eds. C. Amsler, G. Backenstoss, R. Klapisch, C. Leluc, D. Simon and L. Tauscher; Nucl. Science Res. Conf. Ser. 14, p. 19, Harwood Academic Publ. (1988)

7. A. Coc, R. Le Gac, M. de Saint Simon, C. Thibault, F. Touchard, E. Haebel, H. Herr, R. Klapisch, G. Lebee, G. Petrucci, G. Stefanini, A. Schempp, H. Deitinghoff, and F. Botlo-Pilat; in "Low Energy Antiproton Physics, LEAP '90", eds. P. Carlson, A. Kerek, and S. Scilagy; p. 470, World Scientific (1991)

8. J. Eades, D. Hadjukovic, V. Lagomarsino, G. Manuzio, G. Testera, A. Wolf, N. Beverini. R. Poggiani, G. Torelli, L. M. Simons, D. Cauz, A. De Angelis, F. Scuri, F. Waldner, K. Elsener; Proposal CERN/PSCC/89-15, PSCC/P 118 (1989)

9. G. Gabrielse, X. Fei, K. Helmerson, S. L. Rolston, R. Tjoelker, T. A. Trainor, H. Kalinowsky, J. Haas, W. Kells; Phys. Rev. Lett. 57, 2504 (1986)

10. M. Holzscheiter; Physica Scripta 46, 272 (1992)

11. A. Coc, R. Fergeau, C. Thibault, M. de Saint Simon, F. Touchard, E. Haebel, H. Herr, R. Klapisch, G. Lebee, G. Petrucci, G. Stefanini; in "Physics with Antiprotons at LEAR in the ACOL Era"; eds. U. Gastaldi, R. Klapisch, J. M. Richard, J. Tran Thanh Van; p. 675, Editions Frontieres (1985)

12. Memorandum CERN/SPSLC/92/28, SPSLC/M494 P118T Collaboration 1992

13. J. F. Ziegler, J. P. Biersack, U. Littmark; "The Stopping and Range of Ions in Solids"; Pergamon (1985)

14. G. Gabrielse, X. Fei, L. A. Orozco, S. L. Rolston, R. L. Tjoelker, T. A. Trainor, J. Haas, H. Kalinowsky, and W. Kells; Phys. Rev. A40, 481 (1989)

15. S. L. Rolston, G. Gabrielse; Hyper. Inter. 44 (1988) 233

16. N. Beverini, L. Bracci, G. Torelli, V. Lagomarsino, G. Manuzio; Europhysics Lett. 1, 435 (1986)

17. H. G. Dehmelt; Adv. At. Mol. Phys. 5, 109 (1969)

18. G. Gabrielse, L. Haarsma, S. L. Rolston; Int. J. of Mass Spec. Ion Proc. 88, 319 (1988)

19. M. Holzscheiter, et al.; submitted to N IM (1994)

20. G. Graeff, H. Kalinowsky, J. Traut; Z. Physik A297 (1980) 35

21. H. Knudsen, J. F. Reading; Phys. Report 212 (1992) 107

22. E. Uggerhoj; Nucl. Phys. A558 (1993) 655c

23. A. Adamo et al; Nucl. Phys. A558 (1993) 665c

24. E. Uggerhoj et al.; SPSLC 93-28/SPSLC/M523 (1993)

25. T. A. Armstrong, et al., Phys. Rev. C47 (1993) 1957 
26. J. Jastrzebski, H. Daniel, T. von Egidy, A. Gabrowska. Y. S. Kim, W. Kurcewicz. P. Lubinski, G. Riepe, W. Schmid, A. Stolarz, S. Wycech; Nucl. Phys. A558 (1993) 405c

27. B. A. Nikolaus, T. Hoch, D. G. Madland; Phys. Rev. C46 (1992) 1757

28. "The Hydrogen Atom"; eds. G. F. Bassani, M. Inguscio, T. W. Haensch; Springer (1989)

29. G. Budker and A. Skrinsky; Sov. Phys.-Usp. 21, 277 (1978)

30. H. Herr, D. Möhl, and A. Winnacker; in "Proc. 2nd Workshop on Physics with Cooled Low Energy Antiprotons at LEAR", Erice, May 9-16, 1982, (eds. U. Gastaldi and R. Klapisch) p. 659. Plenum, New York 1984

31. R. Neumann, H. Poth, A. Wolf, and A. Winnacker; Z. Phys. A313, 253 (1984)

32. H. Poth; Appl. Phys. A43, 287 (1987)

33. G. Gabrielse, S. L. Rolston, L. Haarsma, and W. Kells; Phys. Lett. A129, 38 (1988)

34. B. I. Deutch, A. S. Jensen, A. Miranda, and G. C. Oades; Proc. 1st Workshop on Antimatter Physics at Low Energies, FNAL, Batavia, IL (1986) ; B. I. Deutch, F. M. Jacobsen, L. H. Andersen, P. Hvelplund, H. Knudsen, M. H. Holzscheiter, M. Chariton, G. Laricchia, Phys. Scrip. T22, 288 (1988)

35. H. Poth; ibid. ref [6], p. 307

36. A. Rich, R. Conti, W. Frieze, D. W. Gidley, H. Griffin,

M. Skalsey, T. Steiger, J. Van House, W. Zheng, and P. Zitzewitz; ibid. ref [6], p321

37. J. W. Humberston, M. Charlton, F. M. Jacobsen, and B. I. Deutch; J. Phys. B20, L25 (1987)

38. B. I. Deutch, L. H. Andersen, P. Hvelplund, F. M. Jacobsen, H. Knudsen, M. H. Holzscheiter, M. Charlton, and G. Laricchia; Hyperfine Int. 44, 271 (1988)

39. M. Charlton; Phys. Lett. A143, 143 (1990)

40. B. I. Deutch, M. Charlton, M. H. Holzscheiter, P. Hvelplund, L. V. Jorgensen, H. Knudsen, G. Laricchia, J. P. Merrison, and M. R. Poulsen; sub. to Hyperfine Interactions (1992)

41. C. T. Munger, M. Mandelkern, J. Schultz, G. Zioulas, T. A. Armstrong, M. A. Hasan, R. A. Lewis, and G. A. Smith; Proposal to Fermilab (1992)

42. Jet Set Collaboration, LEAR/CERN; priv. communication

43. H. Dehmelt, R. Van Dyck, P. Schwinberg, and G. Gabrielse; Bull. Am. Phys. Soc. 24, 757 (1979)

44. T. Szefzick, R. Broeders, M. Dahmen, D. Grzonka, K. Kilian, W. Oelert, K Roehrich, M. Rook, K. Sachs, O. Steinkamp. M. Chanel, P. Lefevre, D. Moehl, G. Molinari; internal report, Jet SET collaboration (1994)

45. G. Gabrielse; priv. communication, also these proceedings.

46. Guo Zhong Li, and G. Werth; Physica Scripta 46, 587 (1992)

47. A. M. Ermolaev, B. H. Brandsen, and C. R. Mandel; Phys. Lett. A25, 44 (1987)

48. A. M. Ermolaev; Hyperfine Int. 44, 375 (1988)

49. P. J. Schultz and K. G. Lynn, Rev. Mod. Phys. 60, 701 (1988)

50. M. Charlton and G. Laricchia; Hyperfine Int. 76 (1992) 97

51. T. J. Murphy, C. M. Surko; Phys. Rev. A46, 5696 (1992) 
52. R. Conti, A. Rich, D. Gidley, M. Skalsey, J. van House, P. W. Zitzewitz, H. Poth, W. Schwab, B. Seligman, M. Woertge, and A. Wolf; Hyp. Int. 44, 201 (1988)

53. G. Gabrielse and B. L. Brown; in "The Hydrogen Atom"; eds. G. F. Bassani, M. Inguscio, and T. W. Hänsch; Springer Verlag Berlin, Heidelberg (1989) B. L. Brown, L. Haarsma, G. Gabrielse, K. Abdulah; Hyp. Int. 73, 193 (1992)

54. A. Kamal et al.; these proceedings

55. K. F. Canter, A. P. Mills, Jr., and S. Berko; Phys. Rev. Lett 33, 7 (1974)

56. A. P. Mills, Jr., Phys. Rev. Lett. 41, 1828 (1978)

57. K. P. Ziock, R. H. Howell, F. Magnotta, R. A. Failor and K. M. Jones; Phys. Rev. Lett 64, 2366 (1990)

58. T. E. Cowan; B. R. Heck, J. H. Hartley, R. H. Howell, R. R. Rohatgi, J. Fajans, R. Gopalan; Hyperfine Int. 76 (1993) 135

59. D. E. Pritchard; Phys. Rev. Lett. 51, 1336 (1983)

60. H. F. Hess, G. P. Kochanski, J. M. Doyle, N. Masuhara, D. Kleppner, and T. Greytak; Phys. Rev. Lett. 59, 672 (1987)

61. D. Kleppner; in "The Hydrogen Atom"; eds. G. F. Bassani, M. Inguscio, and T. W. Hänsch; Springer Verlag Berlin, Heidelberg (1989)

62. J. T. M. Walraven, R. van Roijen, and T. W. Hijmans; in "The Hydrogen Atom"; eds. G. F. Bassani, M. Inguscio, and T. W. Hänsch; Springer Verlag Berlin, Heidelberg (1989)

63. D. Phillips, G. Gabrielse; these proceedings

64. R. J. Hughes, B. I. Deutch; Phys. Rev. Lett. 69 (1992) 578

65. P. Robertson et al;; Phys. Rev. C16 (1977) 1945

66. M. Kasevich and S. Chu; Appl. Phys. B54 (1992) 321

67. T. W. Haensch and C. Zimmermann; Hyp. Int. 76 (1993) 47 
Figure Captions:

Figure 1: General lay-out of the PS200 catching trap set-up. Shown are the magnet dewar with the 6 Tesla magnet and the magnetic field profile, the catching trap, the beam monitor, the gas cell to fine-tune the beam energy, and the scintillation counters to detect antiproton annihilations to measure the incoming beam flux as well as the number of particles released from the trap and annihilating on the microchannel plate detector.

Figure 2: Energy spectrum of approximately 500,000 antiprotons released from the catching trap $500 \mathrm{msec}$ after capture. The horizontal axis represents the energy scale with channel 0 being equivalent to $30 \mathrm{keV}$ and channel 600 approximately $0 \mathrm{eV}$.

Figure 3: Lifetime of antiprotons in the catching trap. The solid line shows the result of an exponential fit to the first 11 data points, yielding a lower limit of the storage time of 30 minutes.

Figure 4: Energy spectra for three different delay times: (a) 8 seconds, (b) 20 seconds, and (c) 40 seconds. The total number of counts (after normalization to the incoming beam intensity) is identical in all three cases.

Figure 5: Shift of the centroid of the energy distribution of the released antiprotons vs. time. An exponential fit to these data yields a cooling time constant of approximately 16 seconds.

Figure 6: Temporal spectrum of protons extracted from a Penning trap. Case (a) is for proper adjustment of the RF drive, case (b) is shows the result of overheating and continuous boil-off inbetween individual extraction pulses.

Figure 7: Partial level diagram for the reaction ${ }_{20}^{48} \mathrm{Ca}_{28}+\overline{\mathrm{p}}(\mathrm{p}) \rightarrow{ }_{19}^{47} \mathrm{~K}_{28} \rightarrow{ }_{20}^{47} \mathrm{Ca}_{27}$, and ${ }_{20}^{48} \mathrm{Ca}_{28}+\overline{\mathrm{p}}(\mathrm{n}) \rightarrow{ }_{20}^{47} \mathrm{Ca}_{27} \rightarrow{ }_{21}^{47} \mathrm{Sc}_{26}$. Shown are the different prompt and delayed decays which can be used for branch identification. Also shown is the final decay to ${ }_{22}^{47} \mathrm{Ti}_{25}$ which may be used for calibration of the total reaction rate. 
Figure Captions:

Figure 1: General lay-out of the PS200 catching trap set-up. Shown are the magnet dewar with the 6 Tesla magnet and the magnetic field profile, the catching trap, the beam monitor, the gas cell to fine-tune the beam energy, and the scintillation counters to detect antiproton annihilations to measure the incoming beam flux as well as the number of particles released from the trap and annihilating on the microchannel plate detector.

Figure 2: Energy spectrum of approximately 500,000 antiprotons released from the catching trap $500 \mathrm{msec}$ after capture. The horizontal axis represents the energy scale with channel 0 being equivalent to $30 \mathrm{keV}$ and channel 600 approximately $0 \mathrm{eV}$.

Figure 3: Lifetime of antiprotons in the catching trap. The solid line shows the result of an exponential fit to the first 11 data points, yielding a lower limit of the storage time of 30 minutes.

Figure 4: Energy spectra for three different delay times: (a) 8 seconds, (b) 20 seconds, and (c) 70 seconds. The total number of counts (after normalization to the incoming beam intensity) is identical in all three cases.

Figure 5: Shift of the centroid of the energy distribution of the released antiprotons vs. time. An exponential fit to these data yields a cooling time constant of approximately 16 seconds.

Figure 6: Temporal spectrum of protons extracted from a Penning trap. Case (a) is for proper adjustment of the RF drive, case (b) is shows the result of overheating and continuous boil-off inbetween individual extraction pulses.

Figure 7: Partial level diagram for the reaction ${ }_{20}^{48} \mathrm{Ca}_{28}+\overline{\mathrm{p}}(\mathrm{p}) \rightarrow{ }_{19}^{47} \mathrm{~K}_{28} \rightarrow{ }_{20}^{47} \mathrm{Ca}_{27}$, and ${ }_{20}^{48} \mathrm{Ca}_{28}+\overline{\mathrm{p}}(\mathrm{n}) \rightarrow{ }_{20}^{47} \mathrm{Ca}_{27} \rightarrow{ }_{21}^{47} \mathrm{Sc}_{26}$. Shown are the different prompt and delayed decays which can be used for branch identification. Also shown is the final decay to ${ }_{22}^{47} \mathrm{Ti}_{25}$ which may be used for calibration of the total reaction rate. 


\section{LEAR CATCHING TRAP SET - UP}
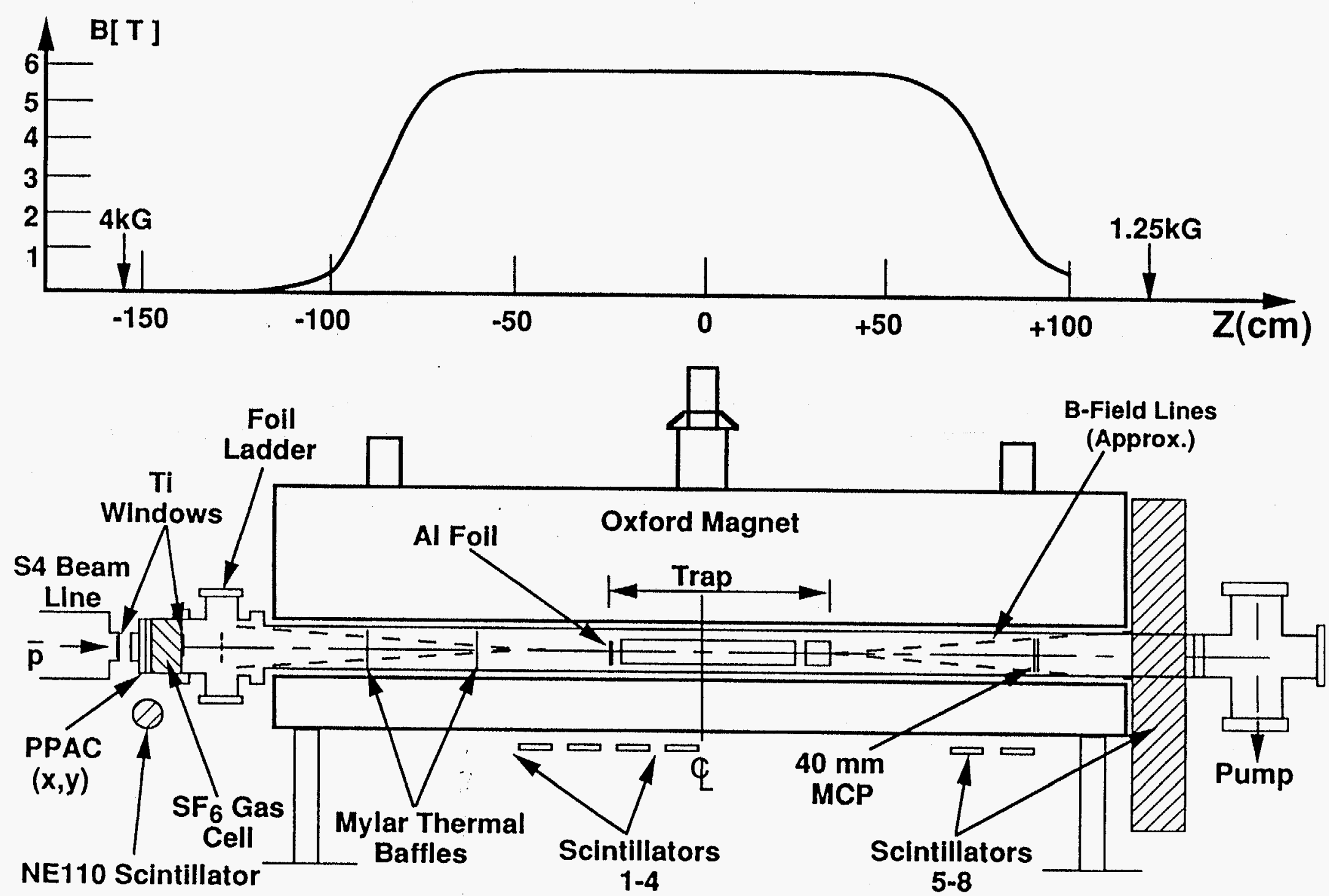


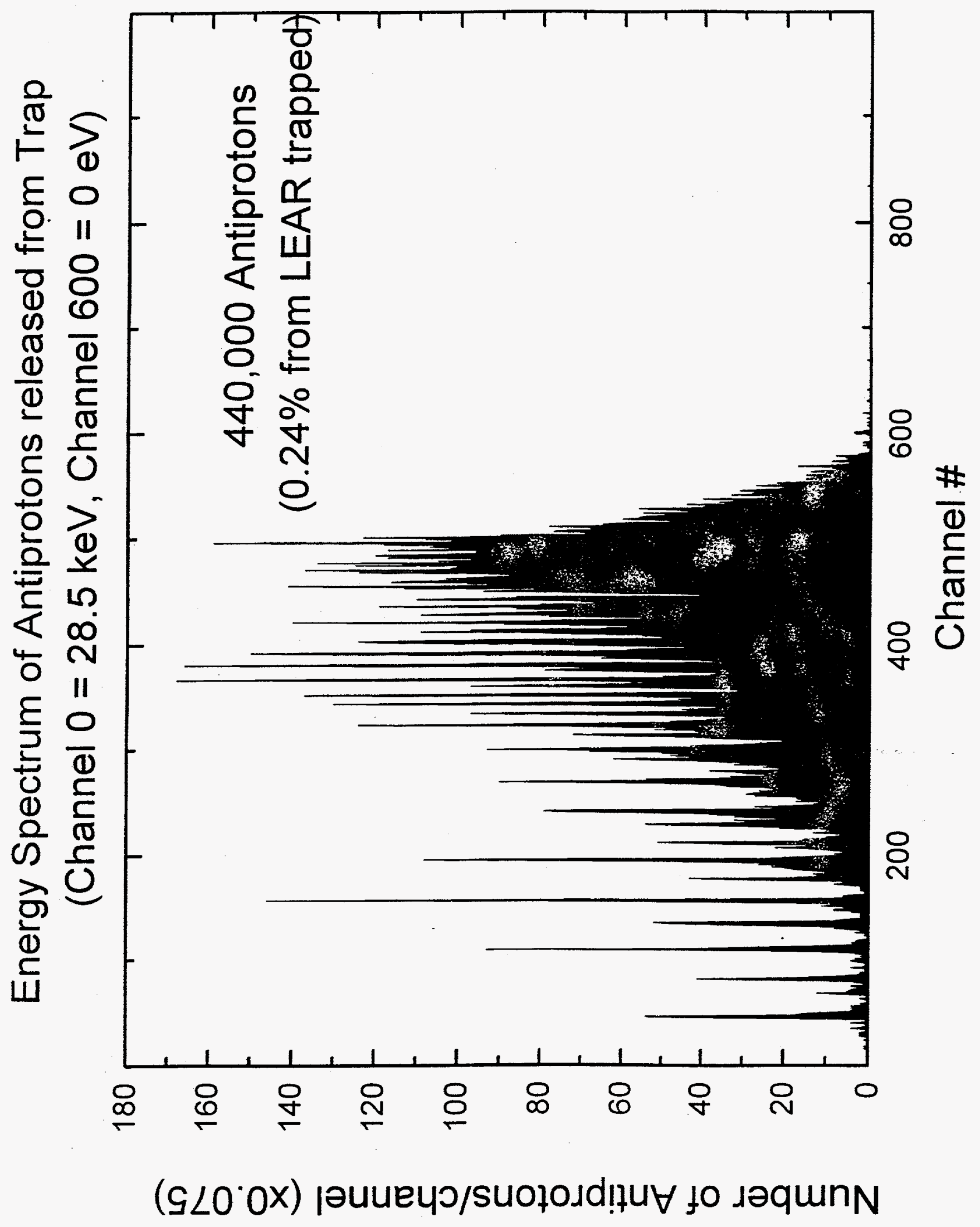




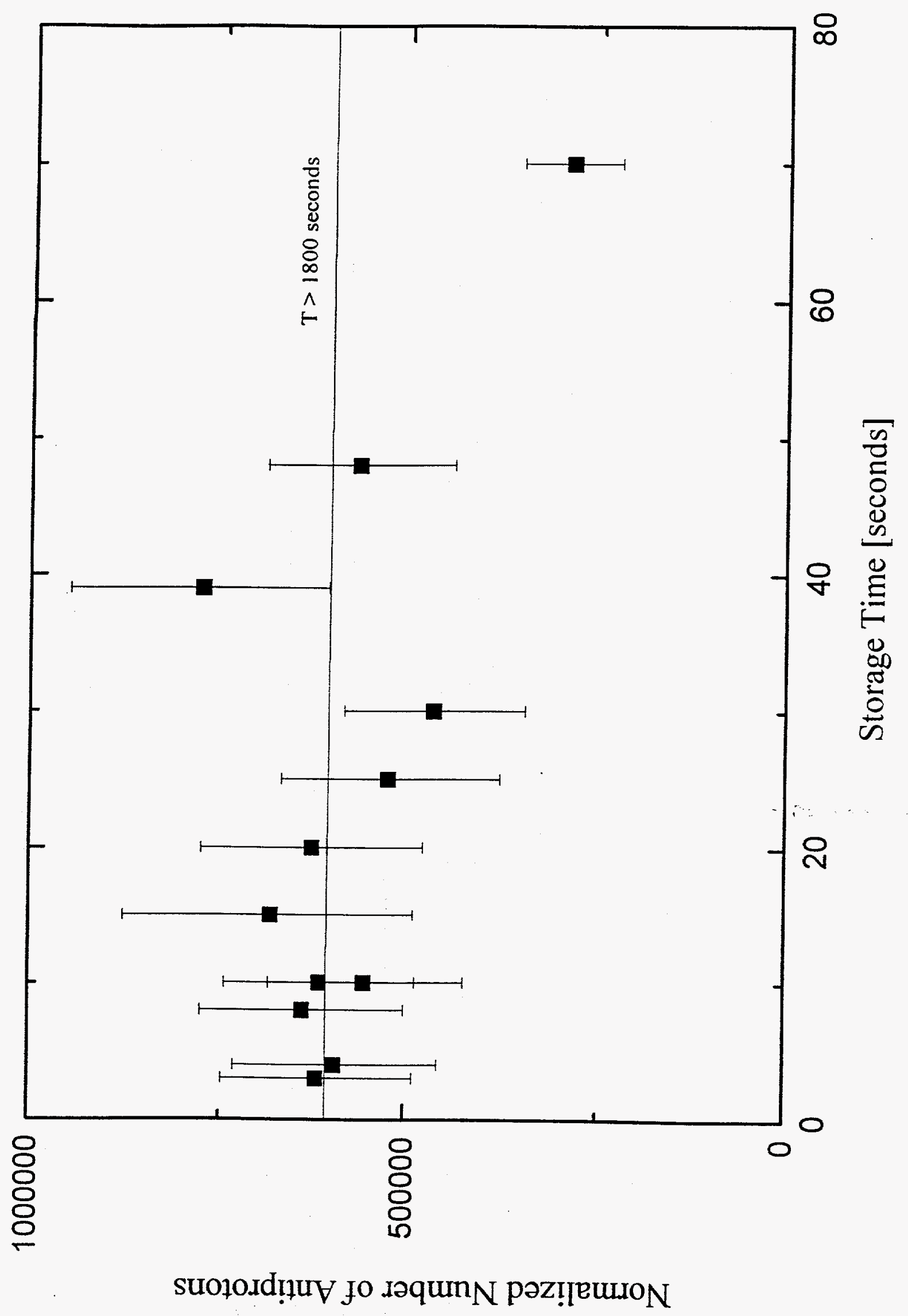



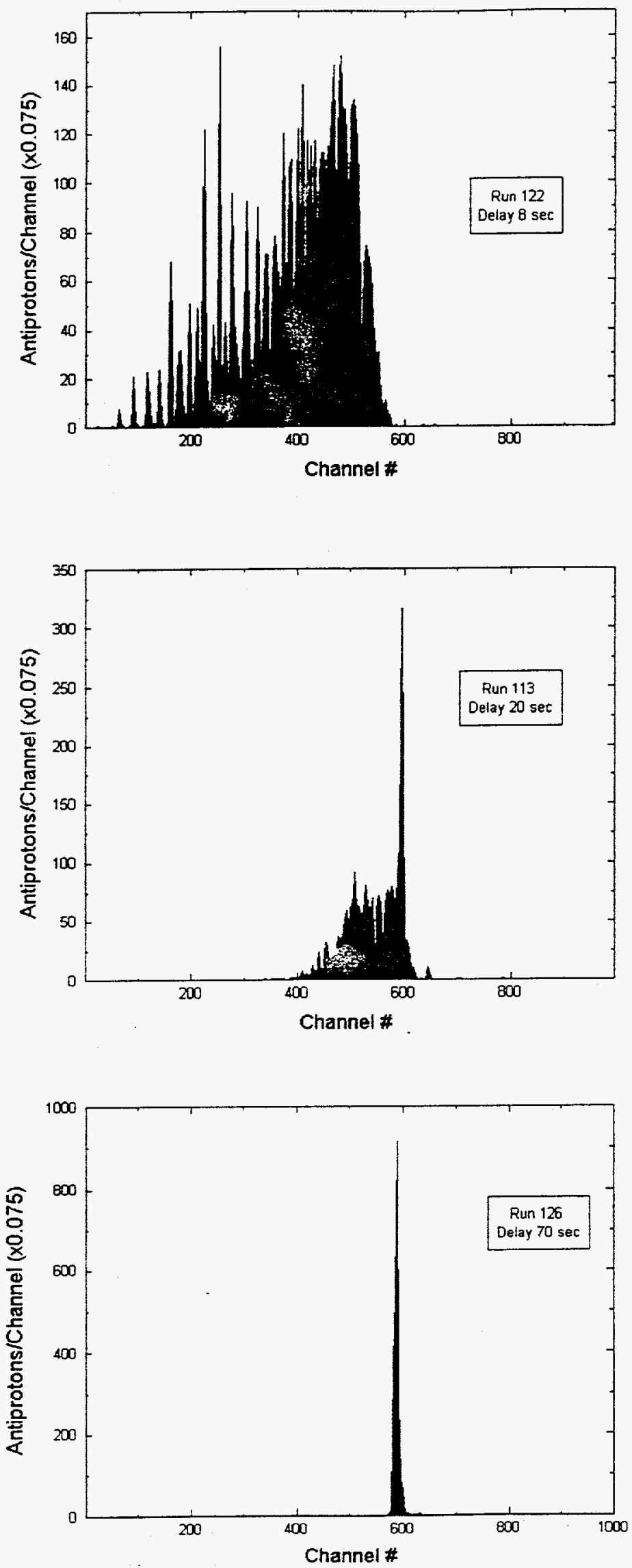


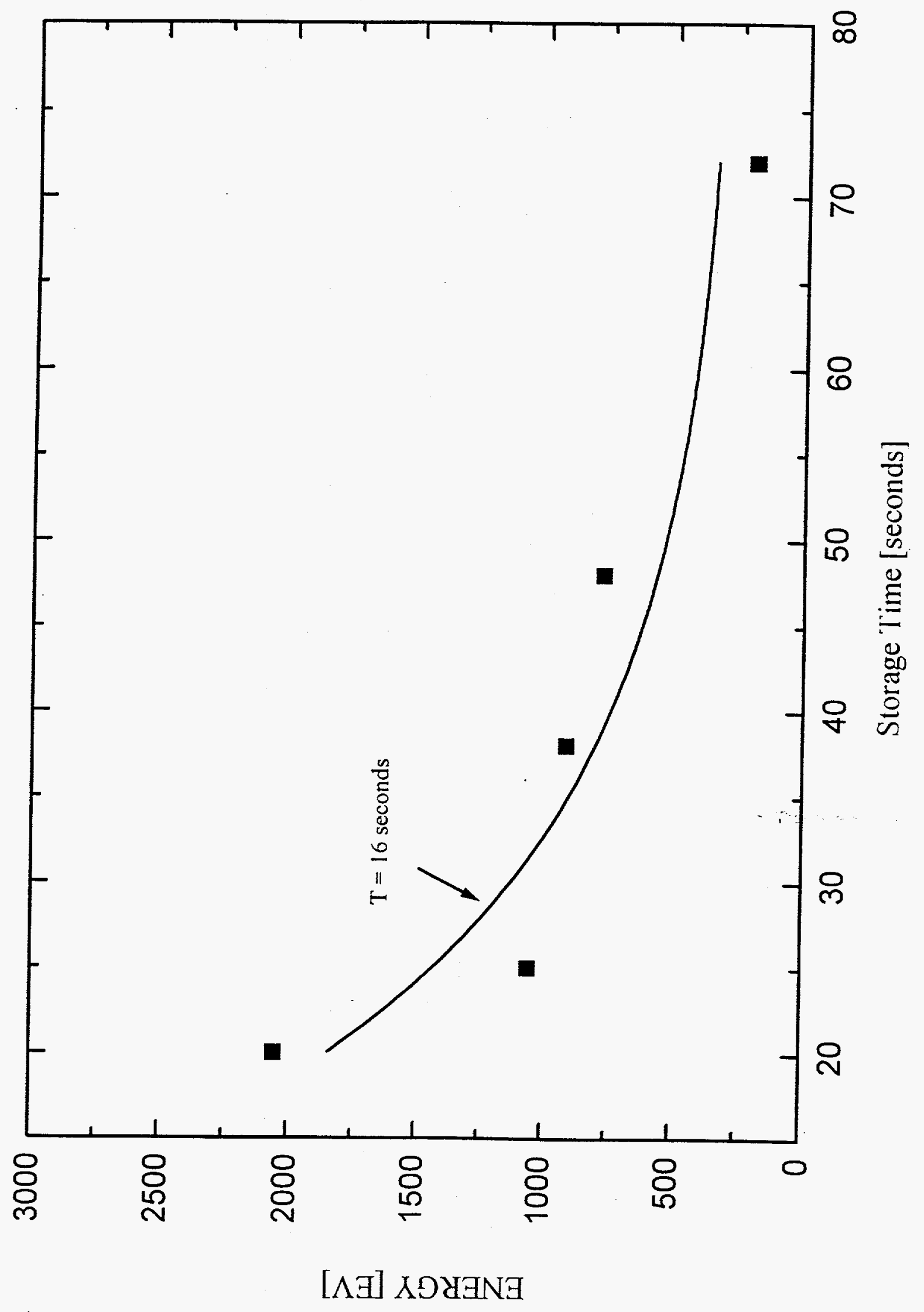




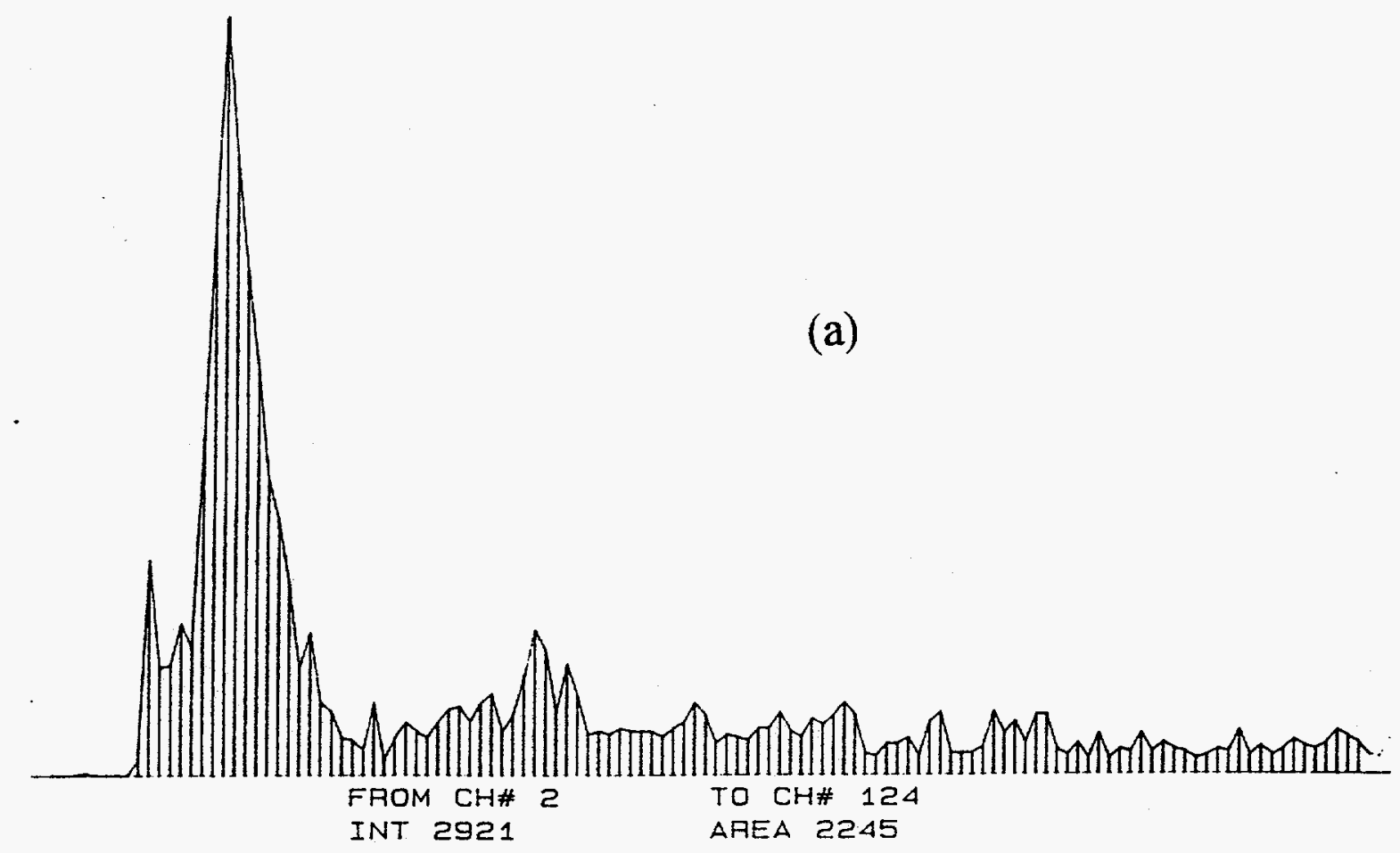

CANBERRA

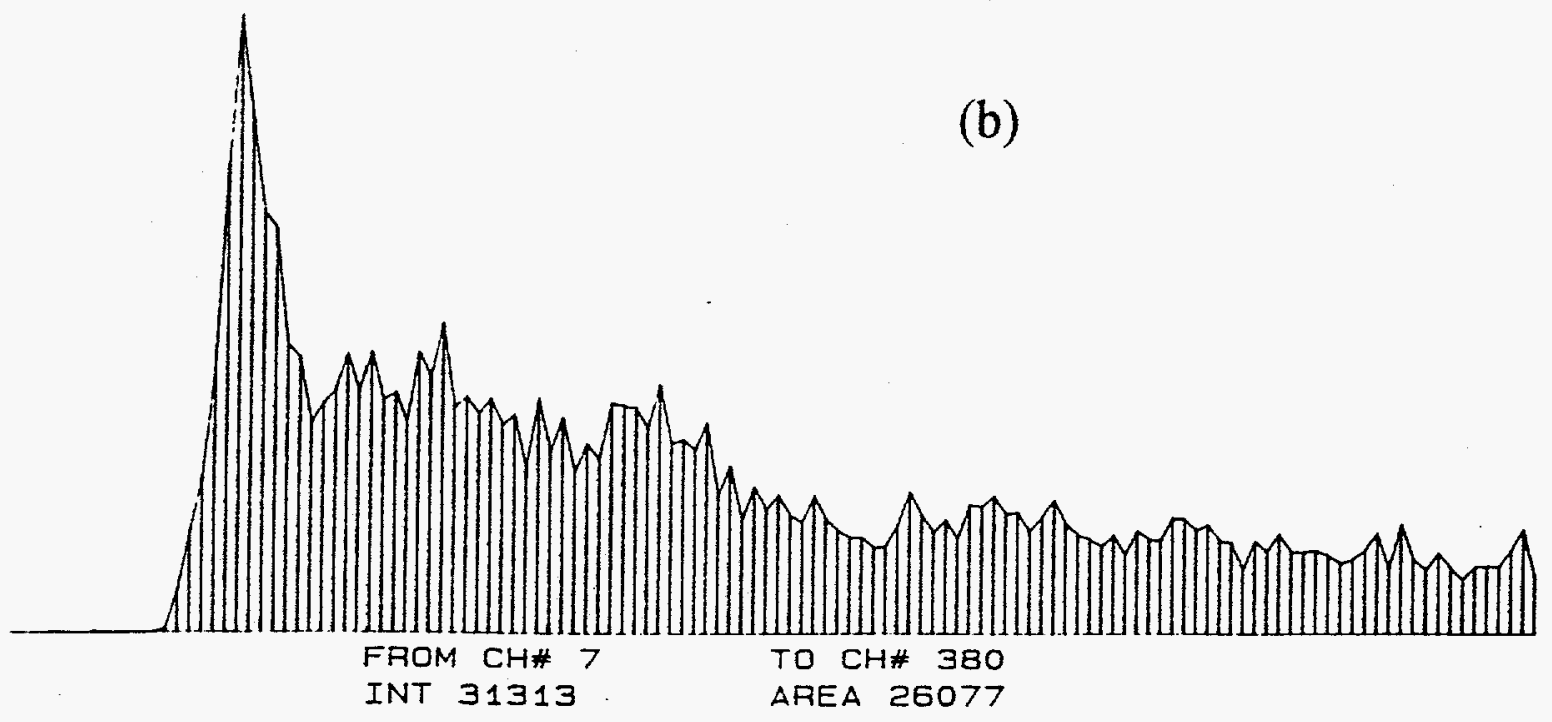




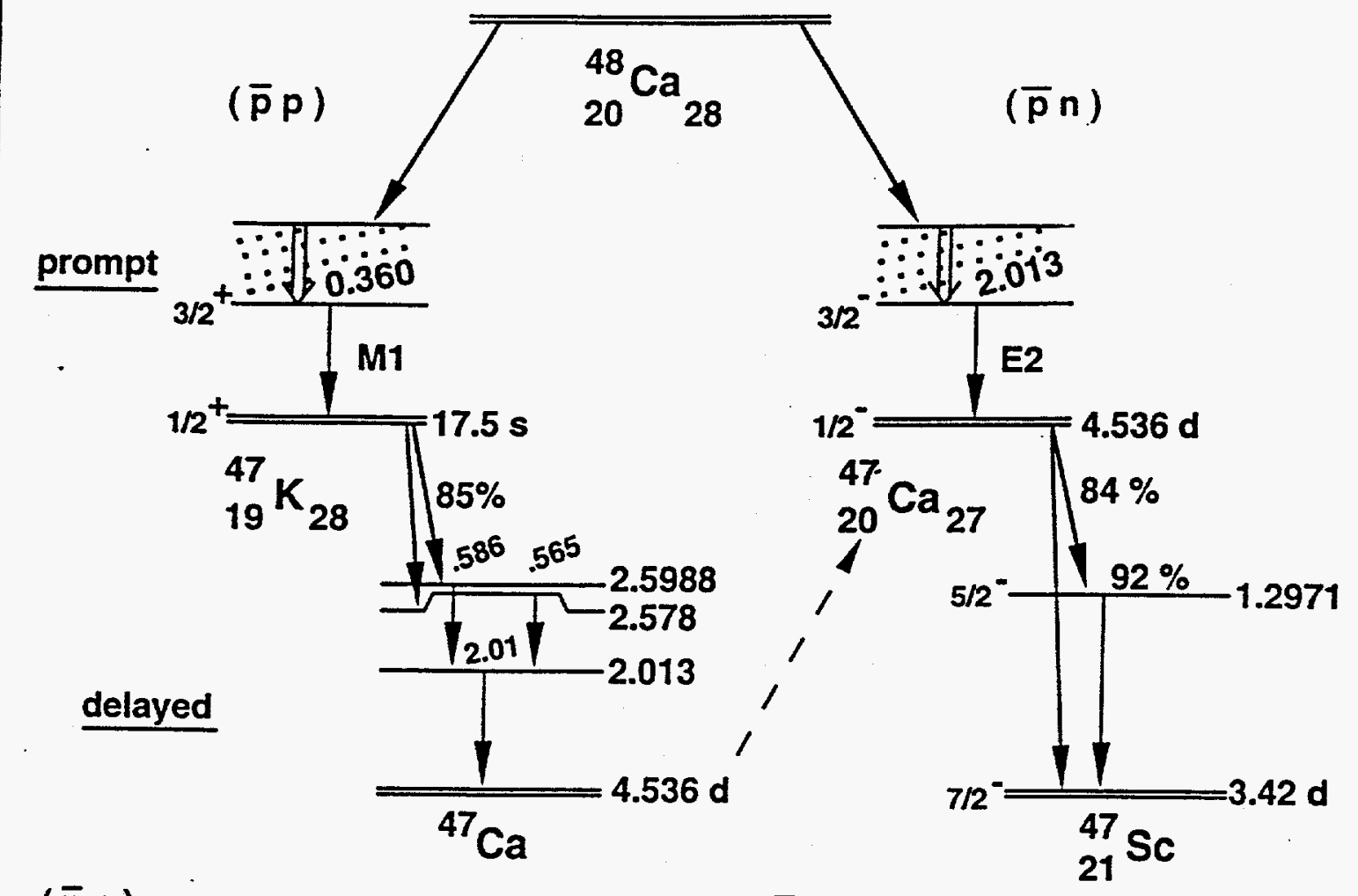

$(\bar{p} p)$

$(\bar{p} n)$

prompt: $360 \mathrm{keV} \gamma ; \sim 3.5 \mathrm{keV}$ X-ray prompt: $2.013 \mathrm{MeV} \gamma ; \sim 3.5 \mathrm{keV}$ X-ray

delayed: $T_{1 / 2} 17.5 \mathrm{~s} \quad 2.013 \mathrm{MeV} \quad$ _delayed: $T_{1 / 2} 4.536 \mathrm{~d} \quad 1.297 \mathrm{MeV}$ delayed: $T_{1 / 2} 4.536 \mathrm{~d} \quad 1.297 \mathrm{MeV}-$ delayed: $T_{1 / 2} 3.42 \mathrm{~d} \quad 0.159 \mathrm{MeV}$

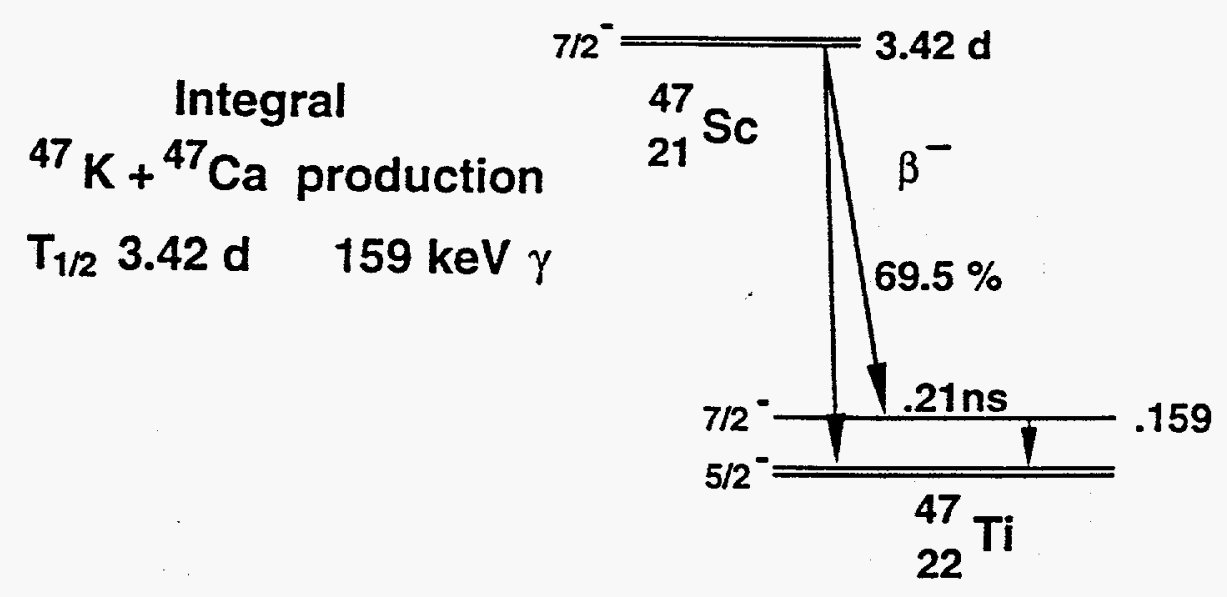

